\title{
Smc5/6 complex regulates Sgs1 recombination functions
}

\author{
Marcelino Bermúdez-López ${ }^{1}$ Luis Aragon ${ }^{1}$
}

Received: 19 July 2016 / Revised: 2 September 2016 / Accepted: 3 September 2016 / Published online: 23 September 2016

(C) The Author(s) 2016. This article is published with open access at Springerlink.com

\begin{abstract}
The family of RecQ helicases is evolutionary conserved from bacteria to humans and play key roles in genome stability. The budding yeast RecQ helicase Sgs1 has been implicated in several key processes during the repair of DNA damage by homologous recombination as part of the STR complex (Sgs1-Top3-Rmi1). Limited information on how is Sgs1 recruited and regulated at sites of damage is available. Recently, we and others have uncover a direct link between the Smc5/6 complex and Sgs1. Most roles of Sgs1 during recombination, including DNA end resection, Holiday junction dissolution, and crossover suppression, are regulated through Mms21-dependent SUMOylation. Smc5/6 first acts as a recruiting platform for STR and then SUMOylates STR components to regulate their function. Importantly, the assembly of STR is totally independent of Smc5/6. Here, we provide a brief overview of STR regulation by Smc5/6.
\end{abstract}

Keywords Smc5/6 complex - Sgs1 - Top3 ·

SUMOylation $\cdot$ Homologous recombination $\cdot$ DNA damage

Communicated by M. Kupiec.

Electronic supplementary material The online version of this article (doi:10.1007/s00294-016-0648-5) contains supplementary material, which is available to authorized users.

Luis Aragon

luis.aragon@csc.mrc.ac.uk

1 Cell Cycle Group, MRC Clinical Sciences Centre, Imperial College, London W12 0NN, UK

\section{Introduction}

DNA damage, originates from endogenous and exogenous sources, and is constantly challenging genome integrity. To overcome this threat, cells have evolved a complex network of intertwined DNA damage repair pathways. Failures in these lifeguard systems compromise genome integrity, which can lead to genome instability, cancer predisposition, and ageing. The RecQ helicases are highly conserved family of proteins from bacteria to humans with critical roles in the maintenance of genome stability over generations. Humans have five distinct RecQ members: BLM, WRN, RECQL1, RECQL4, and RECQL5, whereas only two RecQ-like helicases have been described in budding yeast, Sgs1, and Hrq1 [reviewed in (Bernstein et al. 2010)].

Mutations in BLM cause Bloom syndrome (BS), a genetic disorder characterized by a strong growth deficiency, reduced fertility, diabetes, and cancer predisposition (German et al. 2007). BS cells show increased frequencies of sister chromatid exchanges (SCE), quadriradial chromosome configurations (Chaganti et al. 1974; German et al. 1974), elevated mutation rates and defects in DNA replication expressed as an accumulation of aberrant intermediates (Lonn et al. 1990) and slower replication rates (Hand and German 1975). Over the past decades, many studies have been focused on understanding the functions of BLM during DNA repair. Since the phenotypes of $\operatorname{sgs} 1 \Delta$ cells resemble those observed in BS cells, including replication defects and hyper-recombination (Bernstein et al. 2009), many insights from yeast have been applicable to BLM. Both BLM and Sgs1 form a heteromeric complex with the type IA topoisomerase TOPIII $\alpha$ (Top3 in yeast) (Bennett et al. 2000; Gangloff et al. 1994; Oakley et al. 2002), and the OB-fold RecQ-mediated instability factors, RMI1 and 
RMI2 (Rmi1 in yeast) (Chang et al. 2005; Mullen et al. 2005) and both complexes have been shown to play extensive roles during DNA repair.

\section{Functions of BLM/Sgs1 during homologous recombination}

The BLM-TOPIII $\alpha$-RMI1-RMI2 (BTRR) complex in humans and the Sgs1-Top3-Rmi1 (STR) complex in budding yeast are key players in various processes of DNA metabolism, such as replication, DNA repair, and telomere maintenance. These functions have been extensively reviewed in several journals (Croteau et al. 2014; Manthei and Keck 2013)

Many studies have revealed that BLM/Sgs1 is a central element in the homologous recombination (HR) process, where it carries both pro- and anti-recombinogenic functions. First, BLM/Sgs1, together with DNA2 and EXO1, promotes DSB resection (Gravel et al. 2008; Nimonkar et al. 2011; Nimonkar et al. 2008). Second, it regulates D-loop formation depending on the ADP/ATP-bound state of RAD51. When RAD51 is in its inactive ADP-bound form, BLM can disrupt the RAD51-ssDNA filament preventing the D-loop formation (Bugreev et al. 2007). On the contrary, when RAD51 is in its active ATP-bound form, BLM promotes RAD51 homology search, strand invasion, and, therefore, D-loop formation (Bugreev et al. 2009). Moreover, several studies have shown that BLM/Sgs1 may have a role in synthesis-dependent strand annealing (SDSA) by disrupting D-loops (Bachrati et al. 2006; van Brabant et al. 2000). Third, BTR and STR complexes form the "dissolvasome" which is involved in the processing of late recombination intermediates during the separation of double Holliday junctions (dHJs). Importantly, during the dissolution of dHJs, the helicase activity of BLM/Sgs1 and the topoisomerase activity of TOPIII $\alpha /$ Top 3 are combined to yield products without associated crossovers (Bzymek et al. 2010; Cejka et al. 2010; Ira et al. 2003; Plank et al. 2006; Wu and Hickson 2003). Moreover, the complex promotes the resolution of ultra-fine bridges that can jeopardise chromosome segregation in mitosis (Chan et al. 2007).

BLM/Sgs1 also performs an important role during DNA replication by stabilising stalled forks (Cobb et al. 2003). BLM is present in the advancing replication fork (Alabert et al. 2014), and some results suggests that BLM interacts with FEN1 and thereby assists in the maturation of Okazaki fragments (Bartos et al. 2006; Sharma et al. 2004. Sgs1 is also important for the processing of recombinationdependent structures that appear at damaged forks (Liberi et al. 2005; Mankouri et al. 2007). Because of these roles, BS cells are hypersensitive to drugs that interfere with DNA replication (Davies et al. 2004), and show a higher percentage of fork stalling (Rao et al. 2007). BLM/Sgs1 is involved in replication fork restart after replicative stress (Davies et al. 2007), perhaps, by assisting fork regression (Machwe et al. 2011).

BS cells display telomere defects, such as sister chromatid linkages and telomere loss, which indicate that BLM has a role in processing late-replication intermediate structures that arise during telomere replication (Barefield and Karlseder 2012). It is also involved in the alternative lengthening of telomeres (ALT), a recombination-based mechanism for telomere maintenance. BLM localizes at telomeres specifically in ALT cells and interacts with proteins of the shelterin complex that modulates BLM and WRN unwinding activities on structures that mimic the T-loop in vitro (Lillard-Wetherell et al. 2004; Opresko et al. 2005; Opresko et al. 2002).

\section{Regulation of STR by the Smc5/6 complex}

In recent years, many studies showed that the inactivation of the Smc5/6 complex and sgs $1 \Delta$ cells display similar phenotypes when exposed to DNA damage. They both accumulate recombination-dependent HJs upon replication stress (Ampatzidou et al. 2006; Bermudez-Lopez et al. 2010; Branzei et al. 2006; Liberi et al. 2005; Mankouri et al. 2009; Sollier et al. 2009), and show increased crossover frequencies between sister chromatids (de et al. 2006; Ira et al. 2003; Potts et al. 2006), indicating that their function is probably related. Interestingly, these defects are also seen when the SUMO ligase activity of the Smc5/6 complex is abrogated by the deletion of the C-terminal Siz/ PIAS domain of Mms21/Nse2, a SUMO E3 ligase subunit of Smc5/6 [(Bermudez-Lopez et al. 2010) and Fig. 1].

Using a proteomic screen to look for substrates of Mms21, we identified several subunits within the Smc5/6 complex (Smc5, Smc6, Mms21, Nse3, and Nse4) and two subunits of the STR complex (Sgs1 and Top3) (Bermudez-Lopez et al. 2016). Although Sgs1 had been already described to be SUMOylated in response to DNA damage (Branzei et al. 2006; Lu et al. 2010), neither the SUMO ligase responsible for the modification nor the functional consequences were known. We and others recently demonstrated that the STR complex is able to recognise SUMOylated Smc5/6 complexes at sites of DNA damage (Bermudez-Lopez et al. 2016; Bonner et al. 2016). We proposed that $\mathrm{Smc5/6}$ is attracted to DNA structures that require STR-dependent repair and that upon Smc5/6 engagement, an ATP-dependent remodelling of Smc5/6 drives the activation of the SUMO E3 ligase Mms21, previously demonstrated in (Bermudez-Lopez et al. 2015). As a consequence, SUMOylation of Smc5/6 subunits located at sites of DNA damage would ensue. Crucially, Sgs1 is 


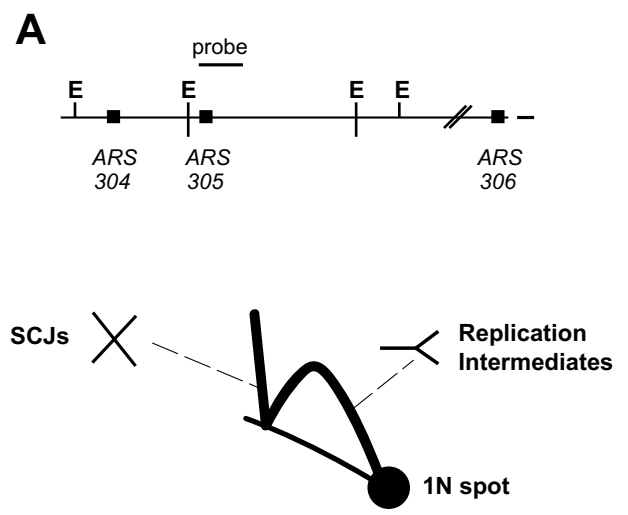

Fig. 1 mms $21 \Delta C$ cells accumulate HJs at damaged forks. Left panel genomic region containing ARS305 origin of replication and probe used for hybridization. Schematic representation of structures visualized by 2D gel electrophoresis. Right panel 2D gel electrophoresis of wild-type and mms $21 \Delta C$ cells. Cells were arrested in G1 with $\alpha$

able to specifically recognise SUMOylated Smc5/6 through two SUMO Interacting Motifs (SIMs) and through this be recruited to these damaged sites (Bermudez-Lopez et al. 2016). Once recruited, STR subunits are SUMOylated in the hands of Mms21, and this results in the activation of the recombinogenic function. Preventing either the recruitment of STR or its SUMOylation causes multiple recombination phenotypes, including decreased DNA end resection, accumulation of recombination-dependent structures at damaged forks and increases in crossover formation during DSB repair (Bermudez-Lopez et al. 2016). Taken together, a two-step role for Smc5/6 in recruiting and activating Sgs1 through SUMOylation seems to fine-tune STR complex functions during recombinational repair.

\section{Assembly of the STR complex in response to DNA damage}

Although the requirement of all three subunits of the STR complex to form a fully functional complex has extensively been described, little is known about how the complex is assembled, particularly in response to DNA damage. During our analysis of STR regulation by Smc5/6, we investigated its effect on STR assembly. Our results revealed that the assembly of STR occurs in two distinct steps that are independent of Smc5/6 function. To analyse STR assembly, we generated strains expressing tagged copies of different STR subunits. First, we investigated the interaction between Top3 and Rmi1 tagging the two proteins (Top3$6 \mathrm{HA}$ and Rmil-9myc). Pull-downs of Rmi1 resulted in a strong co-immunoprecipitation of Top3-6HA (Fig. 2a). This interaction did not require the presence of Sgs1, since

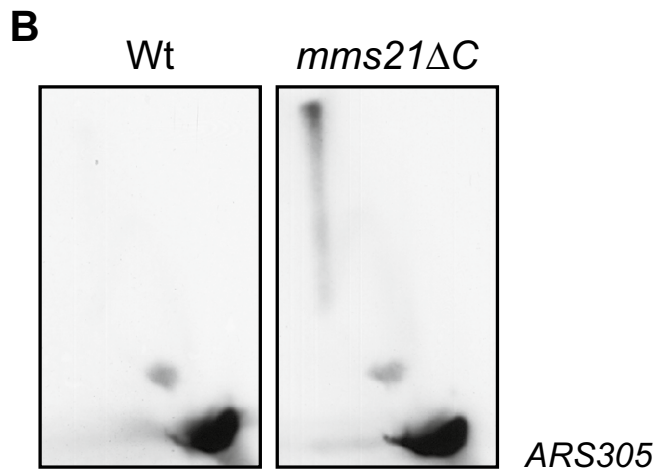

factor. Once arrested, cells were released from G1 arrest into fresh medium containing $0.033 \%$ MMS for $3 \mathrm{~h}$ before samples were taken and processed for $2 \mathrm{D}$ gel. Note the accumulation of SCJs in the $m m s 21 \Delta C$ strain

both proteins co-immunoprecipitated with the same efficiency in sgs 1 null cells (Fig. 2a). This result is consistent with previous work, where Top3 and Rmi1 were shown to form a stable complex even in the absence of Sgs1 (Mullen et al. 2005). Interestingly, this interaction is not affected when cells are subjected to $0.033 \%$ methyl methanesulfonate (MMS) for $2 \mathrm{~h}$, which indicates that the Top3-Rmi1 interaction is independent of DNA damage (Fig. 2b). Next, we decided to explore the Sgs1-Rmi1 and Sgs1-Top3 interactions. In the unchallenged conditions, Sgs1, Top3, and Rmi1 form a heteromeric complex, in which the binding of Rmi1-Top3 subcomplex to Sgs1 is co-dependent (Mullen et al. 2005). Interestingly, both the interaction between Sgs1 and Rmi1 as well as between Sgs1 and Top3 increases when cells are exposed to DNA damage (Fig. 2c, d). Therefore, it is likely that in the unchallenged condition, two types of complexes exist inside cells, one formed by Sgs1Top3-Rmi1 and another formed by Top3-Rmi1, and that the relative amount of Sgs1-Top3-Rmi1 complex, which likely represents functional STR, is increased upon DNA damage.

Based on the fact that under DNA damage conditions, we observed a significant increase in the interaction between Sgs1 and the Smc5/6 complex (Fig. 2e), which occurs in a SUMO-SIM-dependent manner (BermudezLopez et al. 2016), it is tempting to speculate a model, where Smc5/6 recruited to damaged DNA undergoes site-specific SUMOylation to trigger Sgs1 recruitment through Sgs1's SIMs (Fig. 3). Once recruited STR becomes SUMOylated by the Smc5/6 subunit Mms21. This model provides an example of sequential site-specific SUMOylation (first Smc5/6 and then STR) that causes recruitment and activation of complexes involved in DNA damage repair. 

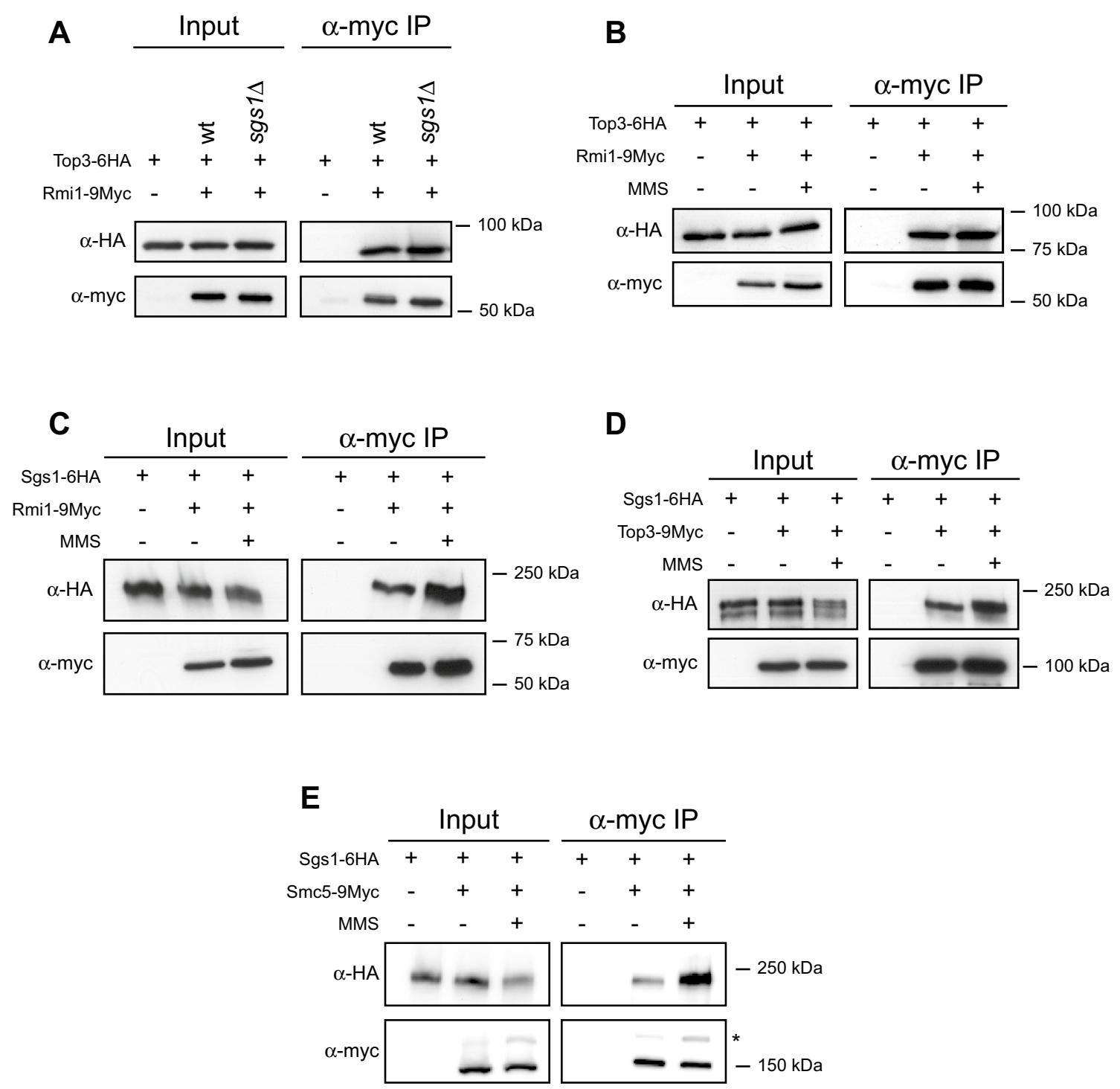

Fig. 2 Assembly of STR complex in response to DNA damage. a Analysis of the Top3-Rmi1 interaction in wild-type and $\operatorname{sgs} 1 \Delta$ cells. b Analysis of the Top3-Rmi1 interaction. c Analysis of the Sgs1Rmi1 interaction. d Analysis of the Top3-Sgs1 interaction. e Analysis of the Sgs1-Smc5 interaction. In $\mathbf{b}-\mathbf{e}$, the culture was split into two

\section{Post-translational modifications of Sgs1}

BLM/Sgs1 has many roles in the repair of DSBs and replication forks (Larsen and Hickson 2013). These roles include both pro- and anti-recombinogenic activities; therefore, its activity must necessarily be tightly controlled to prevent detrimental effects to cells. In the past years, many studies have revealed that post-translational modification of BLM helicases can regulate some of these activities, particularly allowing fine-tuning of its functions. In fact, BLM phosphorylation, ubiquitination, and SUMOylation have been shown to control subcellular localization, interactions, and one half was treated with $0.033 \%$ MMS for $2 \mathrm{~h}$ before collecting them. Rmi1-9myc (a-c), Top3-9myc (d), or Smc5-9myc (e) was immunoprecipitated, and the co-immunoprecipitation of Top3-6HA (a, b) or Sgs1-6HA (c-e) was analysed by Western blot. An asterisk indicates SUMOylated Smc5-9myc

and stability of this helicase [reviewed in (Bohm and Bernstein 2014)]. One of the earliest post-translational modifications described for BLM/Sgs1 was phosphorylation (Davies et al. 2004). The central checkpoint kinase ATM was shown to phosphorylate BLM in response to replication stress. Mutation of BLM phosphor-sites prevented recovery from replication arrests induced by hydroxyurea (HU) but did not affect SCE levels. This led to the proposition that BLM phosphorylation is particularly important for replication stress. Similar to BLM, Sgs1 is phosphorylated upon the activation of the intra S-phase checkpoint by Mec1 (ATR). Phosphorylated Sgs1 contributes synergistically 
Recognition of DNA damage

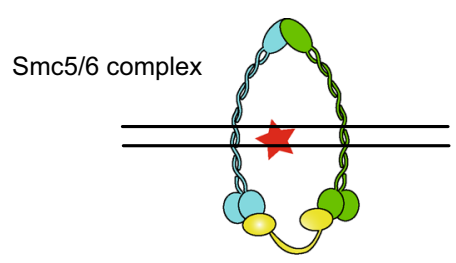

Smc5/6 SUMOylation by Nse2
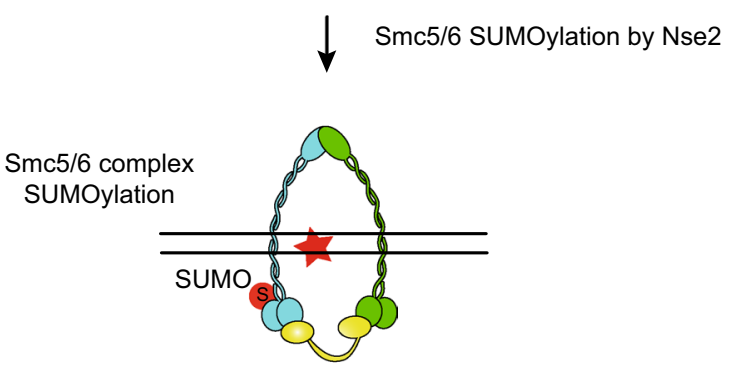

$\downarrow \quad$ Smc5/6 and STR interaction

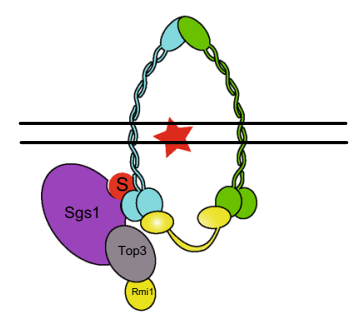

STR SUMOylation by Nse2

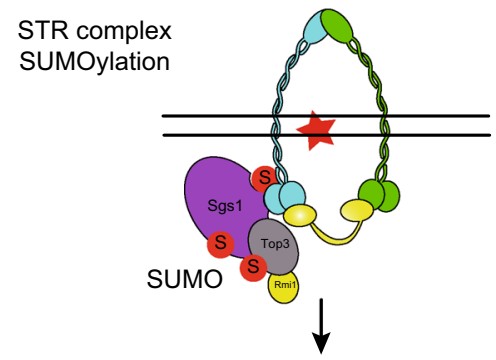

\section{Sgs1 recombination functions}

(DSB end resection, dHJ dissolution, fork repair)

Fig. 3 SUMOylation-dependent recruitment and activation of STR by Smc5/6 complex. Schematic representation of Sgs1's prorecombinogenic functions. Upon DNA damage that requires STR function, we propose that $S \mathrm{mc5} / 6$ is able to recognise suitable DNA substrates for Sgs1. Binding of Smc5/6 to DNA leads the activation of the SUMO E3 ligase Mms21 (Nse2). Nse2 then SUMOylates several subunits of the Smc5/6 complex. Sgs1 (as part of the STR complex) recruitment to these sites occurs via the recognition of SUMOylated Smc5/6 through the SIM domains of Sgs1. Then, Nse2 can SUMOylate Sgs1 and Top3. Nse2-dependent SUMOylation of Sgs1 and Top3 results in the activation of STR functions in recombination to the further activation of $\operatorname{Rad} 53$ (the budding yeast CHK2 homolog), since the N-terminal region of Sgs1 interacts with Rpa70 and recruits Rad53 to stalled forks (Hegnauer et al. 2012).

As described earlier, Sgs1 is SUMOylated and this modification affects many Sgs1 functions (Lu et al. 2010; Bermudez-Lopez et al. 2016; Bonner et al. 2016). Sgs1 contains three lysine residues that lie within SUMO consensus sites ( $\Psi \mathrm{KxE}$ ) at amino-acid positions 175,621 , and 831. Lys621 is the main acceptor site (Lu et al. 2010), but modification also occurs at Lys175 and Lys831. Sgs1 SUMO-deficient cells (sgs 1-3KR) show an accumulation of SCJs when exposed to MMS and show a higher proportion of crossovers and an impaired DNA end resection upon DSBs (BermudezLopez et al. 2016; Bonner et al. 2016). Like Sgs1, BLM is SUMOylated at different positions (Lys317 and Lys331), and SUMO-defective BLM cells also show high levels of SCEs (Eladad et al. 2005). Previous studies revealed that BLM/Sgs1 modification by SUMO leads to changes in the localization of the helicase in response to different DNA damage, but the mechanisms behind the altered localization remain unknown (Eladad et al. 2005; Ouyang et al. 2009). BLM is recruited to PML bodies through SIMs (SUMO interacting motifs), however, the SUMOylated target protein that BLM recognises is unknown (Zhu et al. 2008). In ALT cells, PML bodies contain telomeric DNA, and these types of PML bodies are known as APBs. Interestingly, APBs contain Smc5/6- and Smc5/6-dependent SUMOylation of the shelterin complex which is important to recruit telomeres to PMLs to form APBs (Potts and Yu 2007). It is tempting to speculate that Smc5/6 might promote telomeric recombination in ALT cells by coordinating recruitment of telomeres and BLM to APBs and by activating the pro-recombinogenic role of BLM in an analogous manner to what we have demonstrated for Sgs1 (Bermudez-Lopez et al. 2016; Bonner et al. 2016). Furthermore, sgs1-K621R mutant cells are defective in the production of type II survivors in the absence of telomerase ( $\mathrm{Lu}$ et al. 2010), a yeast mechanism that is thought to be similar to the mammalian ALT pathway for telomere maintenance (Teng and Zakian 1999).Cases of cross talk between SUMOylation and phosphorylation have been demonstrated, for instance, SUMO-regulated phosphorylation has been linked to cell cycle control. However, the relationship between SUMOylation and phosphorylation in the function of Bloom helicases is currently unclear. We predict that interdependence of these two forms of modification is likely to occur, because some Sgs1 functions, particularly in replication forks, are affected by both modifications; nevertheless, future work should address how SUMOylation and phosphorylation fine-tune BLMs function, in which contexts they evoke opposing or similar functional outcomes in the roles played by these helicases. 


\section{Conclusions/summary}

Bloom helicases play key roles relevant to human health; therefore, understanding the molecular mechanisms of Sgs1/BLM function in different contexts is an important challenge. Bloom SUMOylation and its regulation by the Smc5/6 complex provide a new avenue for future studies. Presently, it is clear that Sgs1 is recruited through the recognition of SUMOylated Smc5/6 via SIMs; however, how Sgs1 SUMOylation affects its function remains unknown, one possibility is that SUMOylation modulates STR interactions with other proteins/complexes or DNA substrates; alternatively, this modification might affect helicase activity or protein stability. These are outstanding questions for the future, to gain a comprehensive understanding of STR and its relevance in human health and disease.

\section{Materials and methods}

\section{Yeast strains}

A list of strains used in this study is in supplementary Table 1. Epitope tagging and deletions were performed as described in (Goldstein and McCusker 1999; Janke et al. 2004)

\section{Yeast growth conditions}

Yeast cells were grown in yeast extract peptone media (YEP) supplemented with glucose at $2 \%$ final concentration. To induce DNA damage, cells were treated with $0.033 \%$ MMS for $2 \mathrm{~h}$ before collecting them.

\section{Cell cycle synchronizations}

To synchronize cells in G1, exponentially growing cultures were treated with $\alpha$ factor (Insight Biotechnologies) at a final concentration of $10^{-8} \mathrm{M}$ until $>95 \%$ had been arrested in G1. The release from G1 was conducted by washing cells twice with pre-warmed medium and resuspending them in medium containing $0.1 \mathrm{mg} / \mathrm{ml}$ pronase from Streptomyces griceus (sigma, pronase).

\section{Co-immunoprecipitation}

Co-immunoprecipitation analyses were performed as previously described in (Bermudez-Lopez et al. 2016).

\section{D Gel electrophoresis}

2D Gel electrophoresis analyses were performed as previously described in (Bermudez-Lopez et al. 2016).
Acknowledgments We apologize to all authors whose work we could not cite due to space limitations. We thank the Aragon Laboratory for discussion and critical reading of the manuscript. The work was supported by the intramural programme of the Medical Research Council UK and the Wellcome Trust (100955).

Open Access This article is distributed under the terms of the Creative Commons Attribution 4.0 International License (http://creativecommons.org/licenses/by/4.0/), which permits unrestricted use, distribution, and reproduction in any medium, provided you give appropriate credit to the original author(s) and the source, provide a link to the Creative Commons license, and indicate if changes were made.

\section{References}

Alabert C, Bukowski-Wills JC, Lee SB, Kustatscher G, Nakamura K, de Lima AF, Menard P, Mejlvang J, Rappsilber J, Groth A (2014) Nascent chromatin capture proteomics determines chromatin dynamics during DNA replication and identifies unknown fork components. Nat Cell Biol 16:281-293

Ampatzidou E, Irmisch A, O'Connell MJ, Murray JM (2006) Smc5/6 is required for repair at collapsed replication forks. Mol Cell Biol 26:9387-9401

Bachrati CZ, Borts RH, Hickson ID (2006) Mobile D-loops are a preferred substrate for the Bloom's syndrome helicase. Nucleic Acids Res 34:2269-2279

Barefield C, Karlseder J (2012) The BLM helicase contributes to telomere maintenance through processing of late-replicating intermediate structures. Nucleic Acids Res 40:7358-7367

Bartos JD, Wang W, Pike JE, Bambara RA (2006) Mechanisms by which Bloom protein can disrupt recombination intermediates of Okazaki fragment maturation. J Biol Chem 281:32227-32239

Bennett RJ, Noirot-Gros MF, Wang JC (2000) Interaction between yeast sgs1 helicase and DNA topoisomerase III. J Biol Chem 275:26898-26905

Bermudez-Lopez M, Ceschia A, de PG, Colomina N, Pasero P, Aragon L, Torres-Rosell J (2010) The Smc5/6 complex is required for dissolution of DNA-mediated sister chromatid linkages. Nucleic Acids Res 38:6502-6512

Bermudez-Lopez M, Pocino-Merino I, Sanchez H, Bueno A, Guasch C, Almedawar S, Bru-Virgili S, Gari E, Wyman C, Reverter D, Colomina N, Torres-Rosell J (2015) ATPase-dependent control of the Mms21 SUMO ligase during DNA repair. PLoS Biol 13:e1002089

Bermudez-Lopez M, Villoria MT, Esteras M, Jarmuz A, Torres-Rosell J, Clemente-Blanco A, Aragon L (2016) Sgs1's roles in DNA end resection, $\mathrm{HJ}$ dissolution, and crossover suppression require a two-step SUMO regulation dependent on Smc5/6. Genes Dev 30:1339-1356

Bernstein KA, Shor E, Sunjevaric I, Fumasoni M, Burgess RC, Foiani M, Branzei D, Rothstein R (2009) Sgs1 function in the repair of DNA replication intermediates is separable from its role in homologous recombinational repair. EMBO J 28:915-925

Bernstein KA, Gangloff S, Rothstein R (2010) The RecQ DNA helicases in DNA repair. Annu Rev Genet 44:393-417

Bohm S, Bernstein KA (2014) The role of post-translational modifications in fine-tuning BLM helicase function during DNA repair. DNA Repair (Amst) 22:123-132

Bonner JN, Choi K, Xue X, Torres NP, Szakal B, Wei L, Wan B, Arter M, Matos J, Sung P, Brown GW, Branzei D, Zhao X (2016) Smc5/6 mediated sumoylation of the Sgs1-Top3-Rmi1 complex 
promotes removal of recombination intermediates. Cell Rep. doi:10.1016/j.celrep.2016.06.015

Branzei D, Sollier J, Liberi G, Zhao X, Maeda D, Seki M, Enomoto T, Ohta K, Foiani M (2006) Ubc9- and mms21-mediated sumoylation counteracts recombinogenic events at damaged replication forks. Cell 127:509-522

Bugreev DV, Yu X, Egelman EH, Mazin AV (2007) Novel pro- and anti-recombination activities of the Bloom's syndrome helicase. Genes Dev 21:3085-3094

Bugreev DV, Mazina OM, Mazin AV (2009) Bloom syndrome helicase stimulates RAD51 DNA strand exchange activity through a novel mechanism. J Biol Chem 284:26349-26359

Bzymek M, Thayer NH, Oh SD, Kleckner N, Hunter N (2010) Double Holliday junctions are intermediates of DNA break repair. Nature 464:937-941

Cejka P, Plank JL, Bachrati CZ, Hickson ID, Kowalczykowski SC (2010) Rmil stimulates decatenation of double Holliday junctions during dissolution by Sgs1-Top3. Nat Struct Mol Biol 17:1377-1382

Chaganti RS, Schonberg S, German J (1974) A manyfold increase in sister chromatid exchanges in Bloom's syndrome lymphocytes. Proc Natl Acad Sci USA 71:4508-4512

Chan KL, North PS, Hickson ID (2007) BLM is required for faithful chromosome segregation and its localization defines a class of ultrafine anaphase bridges. EMBO J 26:3397-3409

Chang M, Bellaoui M, Zhang C, Desai R, Morozov P, Delgado-Cruzata L, Rothstein R, Freyer GA, Boone C, Brown GW (2005) RMI1/ NCE4, a suppressor of genome instability, encodes a member of the RecQ helicase/Topo III complex. EMBO J 24:2024-2033

Cobb JA, Bjergbaek L, Shimada K, Frei C, Gasser SM (2003) DNA polymerase stabilization at stalled replication forks requires Mec1 and the RecQ helicase Sgs1. EMBO J 22:4325-4336

Croteau DL, Popuri V, Opresko PL, Bohr VA (2014) Human RecQ helicases in DNA repair, recombination, and replication. Annu Rev Biochem 83:519-552

Davies SL, North PS, Dart A, Lakin ND, Hickson ID (2004) Phosphorylation of the Bloom's syndrome helicase and its role in recovery from S-phase arrest. Mol Cell Biol 24:1279-1291

Davies SL, North PS, Hickson ID (2007) Role for BLM in replication-fork restart and suppression of origin firing after replicative stress. Nat Struct Mol Biol 14:677-679

de Piccoli G, Cortes-Ledesma F, Ira G, Torres-Rosell J, Uhle S, Farmer S, Hwang JY, Machin F, Ceschia A, McAleenan A, Cordon-Preciado V, Clemente-Blanco A, Vilella-Mitjana F, Ullal P, Jarmuz A, Leitao B, Bressan D, Dotiwala F, Papusha A, Zhao X, Myung K, Haber JE, Aguilera A, Aragon L (2006) Smc5-Smc6 mediate DNA double-strand-break repair by promoting sisterchromatid recombination. Nat Cell Biol 8:1032-1034

Eladad S, Ye TZ, Hu P, Leversha M, Beresten S, Matunis MJ, Ellis NA (2005) Intra-nuclear trafficking of the BLM helicase to DNA damage-induced foci is regulated by SUMO modification. Hum Mol Genet 14:1351-1365

Gangloff S, McDonald JP, Bendixen C, Arthur L, Rothstein R (1994) The yeast type I topoisomerase Top3 interacts with Sgs1, a DNA helicase homolog: a potential eukaryotic reverse gyrase. Mol Cell Biol 14:8391-8398

German J, Crippa LP, Bloom D (1974) Bloom's syndrome. III. Analysis of the chromosome aberration characteristic of this disorder. Chromosoma 48:361-366

German J, Sanz MM, Ciocci S, Ye TZ, Ellis NA (2007) Syndromecausing mutations of the BLM gene in persons in the Bloom's Syndrome Registry. Hum Mutat 28:743-753

Goldstein AL, McCusker JH (1999) Three new dominant drug resistance cassettes for gene disruption in Saccharomyces cerevisiae. Yeast 15:1541-1553
Gravel S, Chapman JR, Magill C, Jackson SP (2008) DNA helicases Sgs1 and BLM promote DNA double-strand break resection. Genes Dev 22:2767-2772

Hand R, German J (1975) A retarded rate of DNA chain growth in Bloom's syndrome. Proc Natl Acad Sci USA 72:758-762

Hegnauer AM, Hustedt N, Shimada K, Pike BL, Vogel M, Amsler P, Rubin SM, van LF, Guenole A, van AH, Thoma NH, Gasser SM (2012) An N-terminal acidic region of Sgs1 interacts with Rpa70 and recruits Rad53 kinase to stalled forks. EMBO J 31:3768-3783

Ira G, Malkova A, Liberi G, Foiani M, Haber JE (2003) Srs2 and Sgs1-Top3 suppress crossovers during double-strand break repair in yeast. Cell 115:401-411

Janke C, Magiera MM, Rathfelder N, Taxis C, Reber S, Maekawa H, Moreno-Borchart A, Doenges G, Schwob E, Schiebel E, Knop M (2004) A versatile toolbox for PCR-based tagging of yeast genes: new fluorescent proteins, more markers and promoter substitution cassettes. Yeast 21:947-962

Larsen NB, Hickson ID (2013) RecQ Helicases: conserved Guardians of Genomic Integrity. Adv Exp Med Biol 767:161-184

Liberi G, Maffioletti G, Lucca C, Chiolo I, Baryshnikova A, CottaRamusino C, Lopes M, Pellicioli A, Haber JE, Foiani M (2005) Rad51-dependent DNA structures accumulate at damaged replication forks in sgs 1 mutants defective in the yeast ortholog of BLM RecQ helicase. Genes Dev 19:339-350

Lillard-Wetherell K, Machwe A, Langland GT, Combs KA, Behbehani GK, Schonberg SA, German J, Turchi JJ, Orren DK, Groden $J$ (2004) Association and regulation of the BLM helicase by the telomere proteins TRF1 and TRF2. Hum Mol Genet 13:1919-1932

Lonn U, Lonn S, Nylen U, Winblad G, German J (1990) An abnormal profile of DNA replication intermediates in Bloom's syndrome. Cancer Res 50:3141-3145

Lu CY, Tsai CH, Brill SJ, Teng SC (2010) Sumoylation of the BLM ortholog, Sgs1, promotes telomere-telomere recombination in budding yeast. Nucleic Acids Res 38:488-498

Machwe A, Karale R, Xu X, Liu Y, Orren DK (2011) The Werner and Bloom syndrome proteins help resolve replication blockage by converting (regressed) holliday junctions to functional replication forks. Biochemistry 50:6774-6788

Mankouri HW, Ngo HP, Hickson ID (2007) Shu proteins promote the formation of homologous recombination intermediates that are processed by Sgs1-Rmi1-Top3. Mol Biol Cell 18:4062-4073

Mankouri HW, Ngo HP, Hickson ID (2009) Esc2 and Sgs1 act in functionally distinct branches of the homologous recombination repair pathway in Saccharomyces cerevisiae. Mol Biol Cell 20:1683-1694

Manthei KA, Keck JL (2013) The BLM dissolvasome in DNA replication and repair. Cell Mol Life Sci 70:4067-4084

Mullen JR, Nallaseth FS, Lan YQ, Slagle CE, Brill SJ (2005) Yeast Rmi1/Nce4 controls genome stability as a subunit of the Sgs1Top3 complex. Mol Cell Biol 25:4476-4487

Nimonkar AV, Ozsoy AZ, Genschel J, Modrich P, Kowalczykowski SC (2008) Human exonuclease 1 and BLM helicase interact to resect DNA and initiate DNA repair. Proc Natl Acad Sci USA 105:16906-16911

Nimonkar AV, Genschel J, Kinoshita E, Polaczek P, Campbell JL, Wyman C, Modrich P, Kowalczykowski SC (2011) BLM-DNA2RPA-MRN and EXO1-BLM-RPA-MRN constitute two DNA end resection machineries for human DNA break repair. Genes Dev 25:350-362

Oakley TJ, Goodwin A, Chakraverty RK, Hickson ID (2002) Inactivation of homologous recombination suppresses defects in topoisomerase III-deficient mutants. DNA Repair (Amst) 1:463-482

Opresko PL, von KC, Harrigan J, Hickson ID, Bohr VA, Laine JP (2002) Telomere-binding protein TRF2 binds to and 
stimulates the Werner and Bloom syndrome helicases. J Biol Chem 277:41110-41119

Opresko PL, Mason PA, Podell ER, Lei M, Hickson ID, Cech TR, Bohr VA (2005) POT1 stimulates RecQ helicases WRN and BLM to unwind telomeric DNA substrates. J Biol Chem 280:32069-32080

Ouyang KJ, Woo LL, Zhu J, Huo D, Matunis MJ, Ellis NA (2009) SUMO modification regulates BLM and RAD51 interaction at damaged replication forks. PLoS Biol 7:e1000252

Plank JL, Wu J, Hsieh TS (2006) Topoisomerase IIIalpha and Bloom's helicase can resolve a mobile double Holliday junction substrate through convergent branch migration. Proc Natl Acad Sci USA 103:11118-11123

Potts PR, Yu H (2007) The SMC5/6 complex maintains telomere length in ALT cancer cells through SUMOylation of telomerebinding proteins. Nat Struct Mol Biol 14:581-590

Potts PR, Porteus MH, Yu H (2006) Human SMC5/6 complex promotes sister chromatid homologous recombination by recruiting the SMC1/3 cohesin complex to double-strand breaks. EMBO J 25:3377-3388

Rao VA, Conti C, Guirouilh-Barbat J, Nakamura A, Miao ZH, Davies SL, Sacca B, Hickson ID, Bensimon A, Pommier Y (2007) Endogenous gamma-H2AX-ATM-Chk2 checkpoint activation in
Bloom's syndrome helicase deficient cells is related to DNA replication arrested forks. Mol Cancer Res 5:713-724

Sharma S, Sommers JA, Wu L, Bohr VA, Hickson ID, Brosh RM Jr (2004) Stimulation of flap endonuclease-1 by the Bloom's syndrome protein. J Biol Chem 279:9847-9856

Sollier J, Driscoll R, Castellucci F, Foiani M, Jackson SP, Branzei D (2009) The Saccharomyces cerevisiae Esc2 and Smc5-6 proteins promote sister chromatid junction-mediated intra-S repair. Mol Biol Cell 20:1671-1682

Teng SC, Zakian VA (1999) Telomere-telomere recombination is an efficient bypass pathway for telomere maintenance in Saccharomyces cerevisiae. Mol Cell Biol 19:8083-8093

van Brabant AJ, Ye T, Sanz M, German JL III, Ellis NA, Holloman WK (2000) Binding and melting of D-loops by the Bloom syndrome helicase. Biochemistry 39:14617-14625

Wu L, Hickson ID (2003) The Bloom's syndrome helicase suppresses crossing over during homologous recombination. Nature 426:870-874

Zhu J, Zhu S, Guzzo CM, Ellis NA, Sung KS, Choi CY, Matunis MJ (2008) Small ubiquitin-related modifier (SUMO) binding determines substrate recognition and paralog-selective SUMO modification. J Biol Chem 283:29405-29415 